\title{
El panorama migratorio internacional: Una mirada desde el género
}

\section{The international migration panorama: A gender perspective}

\section{Nombre: \\ Filiación: \\ País:}

Correo:

\author{
Trinidad L. Vicente Torrado ${ }^{(1)}$ \\ Universidad de Deusto \\ España \\ trinidad.vicente@deusto.es
}

\section{Resumen}

En la actualidad las mujeres representan prácticamente la mitad de la población migrante a nivel mundial. Y ello no es una novedad, sino que esta realidad se viene mostrando ya desde hace varias décadas. Con todo, y debido a la invisibilización que las mujeres migrantes han vivido hasta épocas muy recientes, conocemos mucho mejor los factores y dinámicas relativos a la inmigración masculina que a la femenina. Por ello, este artículo se va a centrar en el panorama migratorio internacional actual, con una especial mirada desde la perspectiva de género, tratando de analizar las causas que, tanto desde las sociedades emisoras como desde las receptoras, contribuyen a explicar sus dinámicas de movimiento, sus pautas de asentamiento, sus proyectos migratorios y las consecuencias que de ellos se derivan para sus protagonistas y sus familias. Para ello, se empleará una metodología básicamente cuantitativa, centrada en el análisis de fuentes secundarias, como son las bases de datos de United Nations, la OIM o la OCDE.

\section{Palabras Claves}

Migración internacional, género, flujos migratorios, mujeres migrantes.

\footnotetext{
${ }^{1}$ La autora es Profesora de la Facultad de Ciencias Sociales y Humanas de la Universidad de Deusto e investigadora del Instituto de Derechos Humanos de la Universidad de Deusto. Sus líneas investigativas son: Migraciones internacionales, derechos humanos y género.
} 


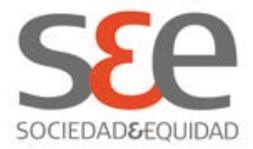

\begin{abstract}
Women represent nearly half of the migrant population at the global level. But this is not a novelty; this has been the reality for the last decades. Despite of this, due to the invisibility that migrant women have experienced until very recently, our knowledge of the factors and dynamics related to the male migrants is much better than the one related to the female migrants. Thus, this paper will focus on the current international migration panorama from a gender perspective. Doing so, I will try to analyze the causes that, from the point of view of the sending and the receiving societies, contribute to explain the movement dynamics, the settlement patterns, the migration projects, and the consequences derived from them for themselves and their families. The methodology used is mainly quantitative, based on the analysis of secondary resources, such as UN, IOM or OECD databases.
\end{abstract}

\title{
Keywords
}

International migration, gender, migration flows, women migrants.

\subsection{La migración internacional y las mujeres. Tendencias recientes}

A lo largo de las tres últimas décadas el número de personas migrantes internacionales se ha duplicado hasta alcanzar en el año 2010 la cifra estimada de 214 millones, aunque su peso relativo en el conjunto de la población mundial se mantiene más o menos constante, en torno al 3,1\% (United Nations, 2012). Y, siguiendo la tendencia de las dos últimas décadas, prácticamente la mitad de estas personas migrantes internacionales son mujeres (49\%).

Las mujeres constituyen la mayoría de las personas migrantes en los países desarrollados $(51,5 \%)$, mientras que su peso relativo es inferior entre la población migrante establecida en las regiones menos desarrolladas (45,3\%). En el año 2010 Europa es el continente con un mayor número de mujeres inmigrantes, seguido de América (y más concretamente Norteamérica), Asia, África y Oceanía. Por otra parte, y a pesar de la creciente diversificación de los países de destino, todavía se registra una importante pauta de concentración de la inmigración femenina: apenas nueve países (EE.UU., Rusia, Alemania, Canadá, Reino Unido, Francia, España, Ucrania e India) acogen a más de la mitad de las mujeres migrantes internacionales. Más aún, una de cada cinco de estas mujeres reside en EE.UU. 


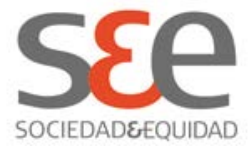

Las migraciones conforman, sin duda, uno de los fenómenos sociales más importantes de nuestros días. $Y$ en este momento de creciente internacionalización, globalización y diversificación de los flujos migratorios, la feminización es, asimismo, otra de sus características frecuentemente señaladas. Ahora bien, a lo largo de los últimos cincuenta años las mujeres migrantes han sido casi tan numerosas como los hombres: en 1960 había 35 millones de migrantes femeninas por 40 millones de migrantes varones y en el año 2010, a pesar del notable aumento de la población migrante, la diferencia entre ambos sexos apenas se reduce, con algo menos de 105 millones de mujeres y 109 millones de hombres viviendo fuera de sus países de origen (United Nations, 2012). La denominada feminización de las migraciones no es, en definitiva, un fenómeno tan novedoso y reciente como a primera vista pudiera parecer (la presencia de las mujeres entre la población migrante internacional tan sólo se ha visto incrementada en dos puntos porcentuales en cuatro décadas), sino que se ha venido gestando desde tiempo antes de que la producción estadística y bibliográfica en materia migratoria se hiciera eco de este asunto. Para entender el concepto de feminización de las migraciones es preciso mirar más allá de los datos cuantitativos (absolutos y/ o relativos) para hacer referencia a la apertura conceptual a la figura de las mujeres migrantes como sujetos social y económicamente activos (Oso, 1998).

Las mujeres, al igual que los hombres, migran siguiendo motivaciones muy diversas, que no pueden reducirse al deseo de seguir a sus esposos, protagonistas del proyecto migratorio. La reagrupación familiar es, sin duda, el motivo principal de muchos proyectos migratorios femeninos, pero también lo es para muchos varones que deciden seguir a sus esposas, pioneras de muchos procesos migratorios internacionales. De hecho, la inmigración femenina supera a la masculina en aproximadamente la mitad de los países a nivel mundial (United Nations, 2012).

A lo largo de las últimas décadas, la migración protagonizada por las mujeres presenta los mismos rasgos de globalización, aceleración y diversificación que los flujos migratorios liderados por los hombres. Pero a día de hoy se conocen mucho mejor las características y los factores relativos a la migración masculina que a la femenina. Por ello, en este artículo se plantea un repaso de los principales flujos y stocks migratorios internacionales desde una perspectiva de género, organizados por continentes. Comenzaremos con Oceanía, el continente que cuenta con una menor presencia de inmigración, para concluir con Europa, el continente que registra el mayor número de inmigrantes internacionales en general, y mujeres inmigrantes en particular. Y todo ello con el objeto de plantear una reflexión inicial en torno a la enorme complejidad de las migraciones internacionales desde una perspectiva de género; un marco general que habrá de ser completado con análisis más específicos, centrados en 


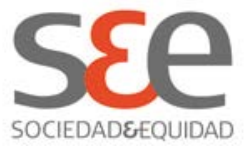

un determinado contexto, colectivo o situación, de modo que nos permitan seguir profundizando en la comprensión de la compleja realidad migratoria actual.

\subsection{Migración internacional en Oceanía}

Oceanía es el continente con un menor número de migrantes internacionales, aunque en algunos países de destino -como Australia y Nueva Zelanda- su peso relativo es muy importante, llegando a representar el $22 \%$ de la población total en cada uno de ellos. Australia, tradicional país de inmigración procedente del continente europeo, recibe en la actualidad un notable contingente de inmigración procedente de Asia y de sus países vecinos de Oceanía (OIM, 2011: 86-88).

Tanto en Australia como en Nueva Zelanda, la migración femenina supera a la masculina, así sea por escaso margen (51,4 y 52,4\% respectivamente) (United Nations, 2012). Precisamente el mayor flujo migratorio desde Asia es el que más ha contribuido a la feminización de la población migrante en esta parte del mundo desde la década de los ochenta (Hugo, 1994). La población migrante procedente de otras áreas es, en cambio, mayoritariamente masculina (Ryan, 2002).

\subsection{Migración internacional en África}

De los 30 millones de personas africanas que residen en un país diferente al de nacimiento (aproximadamente el $3 \%$ de la población), la mitad sigue viviendo en el continente africano. Con la única excepción de la emigración del Magreb (que en el $90 \%$ de los casos se dirige a Europa), podemos señalar que la emigración africana es fundamentalmente una emigración intraregional: dos terceras partes de la población que ha dejado su país en el África Subsahariana reside en otro país de la región, como Burkina Faso, Kenia o Sudáfrica (OIM, 2011: 68). Más concretamente, el $70 \%$ de las personas que emigra al extranjero en el África Occidental lo hace dentro de la región, al igual que el $66 \%$ de quienes lo hacen en el África Meridional y el 52\% en el África Oriental.

Las medidas de ajuste macroeconómicas y el aumento de la pobreza en muchos países africanos, la creciente desertificación y las crisis alimentarias cíclicas o los conflictos armados que todavía sacuden el continente africano, están provocando un flujo migratorio constante, tanto femenino como masculino, cualificado y no cualificado, en busca de mejores oportunidades de vida, tanto dentro del propio continente como fuera de él (en este caso especialmente hacia Europa, EE. UU. o Canadá).

En los países del África Subsahariana la proporción de mujeres inmigrantes ha crecido de forma paralela al incremento en el número de 


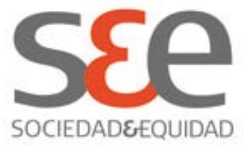

personas refugiadas en el continente (Zlotnik, 2005). Y aunque todavía a día de hoy hay más países en los que los hombres de origen extranjero superan a las mujeres en la misma situación que a la inversa, y a pesar de que ningún país africano se coloca entre los primeros con mayor número de mujeres migrantes internacionales, la inmigración femenina en el África Subsahariana representa un $47 \%$ del total; un porcentaje tan sólo ligeramente inferior al registrado a nivel mundial. Más aún, en países como Angola, Guinea, R.D. del Congo, Níger, Ruanda o Burundi la mayoría de la población inmigrante es femenina, lo que da cuenta de la creciente inmigración autónoma de mujeres en este área geográfica, así sea de forma voluntaria o forzada. Asimismo, otros flujos procedentes de estas áreas de origen se encuentran claramente feminizados, como la migración de Cabo Verde hacia Italia, compuesta en un $85 \%$ por mujeres (UN-UNFPA, 2006).

La contracción de las oportunidades de empleo para los hombres y el aumento de la demanda en sectores típicamente femeninos, como el sanitario, han supuesto un incremento de la migración femenina tanto interna como internacional como una estrategia de supervivencia familiar (Adepoju, 2002).

La feminización de la pobreza en África está cambiando el perfil migratorio tradicional desde este continente. Si antes eran los hombres quienes emigraban a larga distancia, dejando a las mujeres y a la prole en el país de origen, cada vez está adquiriendo mayor importancia la emigración femenina interna e internacional de carácter autónomo en busca de un trabajo remunerado y de mejores oportunidades económicas, y ya no sólo con el objetivo de reunirse con el esposo u otros familiares. En aquellos países en los que la globalización ha venido acompañada de unos mayores índices de pobreza, dejando a las mujeres con limitados derechos económicos, sociales y políticos, la emigración internacional se presenta como una de las escasas alternativas (United Nations, 2006).

Entre estas mujeres que inician un proyecto migratorio internacional podemos encontrar personas no cualificadas, pero también una cantidad importante de migrantes cualificadas, dados los mayores niveles educativos alcanzados en los últimos años por las mujeres en algunos países africanos. Así, doctoras y enfermeras han emigrado desde Nigeria a Arabia Saudí, mientras que desde Ghana, Sudáfrica y Zimbawe estas profesionales se han dirigido principalmente hacia el Reino Unido, Estados Unidos y Canadá en busca de un futuro más próspero (Adjei, 2006). Francia, por su parte, también ha atraído una cantidad creciente de mujeres cualificadas procedentes de zonas urbanas de Senegal (UN-UNFPA, 2006).

Este importante flujo migratorio femenino internacional de personal cualificado (enfermeras, doctoras y profesoras principalmente) está suponiendo 


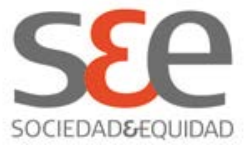

la pérdida de un considerable número de profesionales, poniendo en cuestión incluso la capacidad de estos países africanos para formar a otras nuevas profesionales. Más aún, muchas de estas mujeres van a sufrir un proceso de descualificación, al no obtener trabajos en el extranjero acordes a su nivel de formación, sino de inferior categoría, y primar la consecución de ingresos económicos por encima del estatus social (Massey, et. al., 1993). Pero a pesar de todo ello, la fuga de personal cualificado femenino todavía no ha despertado la atención suficiente desde el ámbito académico o político (Adjei, 2006).

En cuanto al área del Magreb, Egipto y Marruecos son, seguidos a cierta distancia por Sudán y Argelia, los países con mayor emigración internacional. Llama la atención, además, que la población emigrante de Marruecos, Argelia y Túnez se dirija principalmente hacia las regiones más desarrolladas (concretamente hacia Europa occidental y del sur), mientras que la emigración de Egipto, Sudán, Libia y el Sáhara Occidental se orienta sobre todo hacia regiones menos desarrolladas. Así, la emigración egipcia tiene su destino principal en los países del Golfo Pérsico, destino que es compartido con los países del Este africano en el caso de la población sudanesa. La población libia, en cambio, emigra fundamentalmente a Bangladesh, mientras que las personas originarias del Sáhara Occidental se quedan en otro país de la región: Argelia.

Los países del Norte de África son más emisores que receptores de población migrante, tanto masculina como femenina. En todo caso, los varones inmigrantes que residen en esta área geográfica superan a las mujeres por amplio margen ( $57 \%$ y $43 \%$ respectivamente). En ningún país del Magreb se registra un mayor número de mujeres inmigrantes que de hombres (United Nations, 2012). Y, de igual manera, la emigración desde esta región, tanto con destino principal hacia Europa como hacia los países del Golfo Pérsico, está liderada fundamentalmente por los hombres. Y es que en la región de los países árabes, las normas socioculturales siguen limitando la movilidad femenina (UNUNFPA, 2006). Esto no obstante, es reseñable que Marruecos es el país africano que lidera el ranking de emigración femenina, siendo Francia su principal país de destino.

\subsection{Migración internacional en Asia}

Asia es el segundo continente con un mayor stock de población inmigrante internacional (61 millones de personas), tan sólo por detrás del continente europeo (con casi 70 millones). El peso relativo de la inmigración femenina en Asia $(44,6 \%)$ es, en cambio, el más bajo en comparación con el registrado en el resto de continentes.

Según datos del año 2010, cinco de los diez principales países emisores de emigrantes a nivel mundial son asiáticos: Bangladesh, China, Filipinas, India 


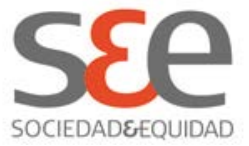

y Pakistán. La migración intracontinental a países como Singapur, Malasia, Hong Kong y la República de Corea es importante, aunque lo es aún más la dirigida hacia los países del Golfo Pérsico. Así lo pone de manifiesto también el hecho de que ocho de los diez países con mayor porcentaje de población inmigrante internacional en relación con su población nativa se ubiquen en la región del Oriente Medio: Arabia Saudí, Bahréin, Emiratos Árabes Unidos, Israel, J ordania, Kuwait, Omán y Qatar (OIM, 2011).

En Asia se da una destacada competencia entre los países emisores de personas emigrantes. India y Pakistán, por ejemplo, han sido tradicionalmente países de emigración masculina hacia el Golfo, siendo en parte desplazados posteriormente por Bangladesh; país cuyos ciudadanos comenzaron a ofrecer trabajo por menores salarios. A comienzos de la década de los ochenta otros países como Sri Lanka e Indonesia también quisieron entrar en este mercado internacional asiático, optando por los sectores laborales femeninos, al encontrar menores dificultades a su incorporación que en los típicamente masculinos. Ya en los años noventa, la población de Nepal también comenzó a emigrar de forma más intensa, desplazando a los trabajadores de Bangladesh al aceptar salarios aún más bajos.

Y muchos países asiáticos receptores, por su parte, también han preferido la rotación de su mano de obra internacional. Así lo muestra claramente el caso de Malasia: este país recibió un gran número de personas trabajadoras, hombres y mujeres, procedentes de Indonesia, con anterioridad a los años noventa. Durante la primera mitad de la última década del siglo XX optó, en cambio, por la inmigración procedente de Bangladesh, prefiriendo posteriormente a la población de origen nepalí. Y desde el año 2006 ha cerrado nuevamente la puerta a la población procedente de este último país -Nepalpara reabrirla a las personas de Bangladesh (Siddiqui, 2008).

La creciente presencia de mujeres en las migraciones internacionales originarias de Asia es, por otra parte, un dato destacable. En algunos países de este continente las mujeres emigrantes conforman una clara mayoría, como en el caso de Filipinas, Sri Lanka, e Indonesia (Oishi, 2005). Las mujeres constituyen el $70 \%$ de personas emigrantes en Filipinas, el primer país del mundo exportador de mano de obra, con destinos situados tanto dentro como fuera del continente asiático. En Sri Lanka, las mujeres constituyen el 65\% del total de emigrantes laborales, siendo sus destinos principales Arabia Saudita, los Emiratos Árabes Unidos y Kuwait. Y en el caso de Indonesia, la emigración femenina también conforma la mayoría, dirigiéndose fundamentalmente al sector del trabajo doméstico del Oriente Medio, Malasia y Singapur (Engle, 2004; UN-UNFPA, 2006). Tailandia o Birmania son también países de emigración femenina, aunque sus fluj os presentan un carácter mucho menos regular. Otros 


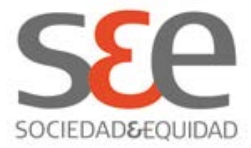

países como Nepal, Bangladesh, India o Pakistán, en cambio, exhiben porcentajes muy reducidos de emigración femenina, seguramente como resultado de las estrictas normas impuestas a su salida, debido a que en estos países la emigración es considerada como muy peligrosa para sus ciudadanas. A pesar de ello, algunas mujeres continúan emigrando, frecuentemente de forma clandestina, asumiendo mayores riesgos y abonando el terreno para las mafias dedicadas al tráfico de seres humanos (Asis, 2005).

Buena parte de la emigración laboral asiática a gran escala, en especial hacia los países del Golfo Pérsico, ha sido masculina, en respuesta a la creciente oferta de empleo en el sector de la construcción. Ahora bien, un pequeño segmento de mujeres emigrantes también ha participado tradicionalmente en estos flujos, ocupando puestos como doctoras, enfermeras y profesoras (Siddiqui, 2008: 4). Esto no obstante, la migración femenina asiática ha crecido de forma muy notable en las últimas décadas en respuesta a los cambios en el mercado de trabajo, que presenta una creciente demanda no sólo de personal sanitario y docente, sino también de empleadas en industrias manufactureras (especialmente en fábricas dedicadas al ensamblaje electrónico y a la confección de ropa), limpiadoras, trabajadoras domésticas, etc. (Chant y Radcliffe, 1992). De igual manera, las crecientes ofertas laborales en el sector del ocio y del "espectáculo" en el caso de Japón, y más recientemente de Corea del Sur, han animado a un creciente número de mujeres, sobre todo filipinas, a iniciar un proyecto migratorio internacional a partir de la década de los años ochenta (Parreñas, 2010). La creciente demanda de "trabajadoras del sexo" en países como la India (cuyas ciudades son abrumadoramente masculinas) 0 en otros países como Tailandia, Singapur 0 Malasia, con un creciente "turismo sexual", está provocando asimismo una creciente migración femenina en Asia, que también se dirige con los mismos fines laborales hacia algunos países europeos ${ }^{2}$ (Skrobanek, et.al., 1997).

La demanda de profesionales de la salud tampoco ha quedado limitada a los países del Golfo Pérsico, sino que también es muy importante su reclamo

\footnotetext{
${ }^{2}$ La trata de personas con fines de explotación sexual conforma la tercera fuente de ganancias para el crimen organizado, después del narcotráfico y la venta ilegal de armas (UN-UNFPA, 2006: 44). Esto no obstante, la mayoría de las mujeres migrantes no son víctimas de este delito ni ejercen la prostitución de manera forzada. Dato a tener en cuenta puesto que el excesivo énfasis en la tríada migración-trata de personasprostitución intensifica la situación de víctima de muchas mujeres migrantes que inician su proyecto en solitario, y que son representadas como personas vulnerables, "sujetos frágiles y débiles", incapaces de decidir y actuar de forma independiente; una visión pasiva de su participación en los movimientos migratorios que va a conformar para ellas otra forma de estigmatización y segregación social (Oishi, 2005; Juliano, 2002).
} 


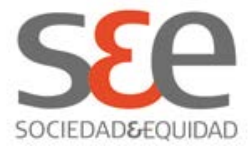

desde algunos países desarrollados (como EE.UU.), e incluso desde algunos países del Sudeste asiático (como Singapur, Tailandia o Malasia); países estos últimos que han implementado servicios de salud de gran calidad con el objeto de atraer a un creciente turismo sanitario. Los países que exportan un mayor número de enfermeras tanto hacia estos otros países asiáticos como hacia Occidente son Filipinas, Sri Lanka e India.

Por otra parte, desde la década de los noventa también es creciente el número de personas, especialmente mujeres, que son objeto de tráfico y trata de personas $^{3}$ y que forman parte del significativo colectivo de población migrante en situación irregular en Asia. Por ello, y por la invisibilidad que también viven las mujeres ubicadas en el servicio doméstico, lo que las coloca en una posición de especial vulnerabilidad y desamparo ante situaciones de explotación laboral -e incluso de violencia de género- en el puesto de trabajo, algunos países de origen (como, por ej. Filipinas o Singapur) han establecido mecanismos legislativos, programas de formación y de orientación previos a la partida, agencias para facilitar la contratación en origen, etc. con el objeto de promover una mayor protección de sus derechos como personas migrantes (Constable, 1997). A día de hoy la protección de las trabajadoras migrantes sigue siendo, no obstante, un reto importante, ya que las medidas tomadas todavía no parecen ser suficientes para el logro del objetivo a alcanzar, como tampoco lo es la escasa cooperación mostrada en general por los países de destino (Huang y Yeoh, 2003; Asis, 2005).

La migración internacional por matrimonio es otra característica destacable entre las mujeres asiáticas. Si en la década de los setenta los matrimonios internacionales tenían lugar preferentemente entre un hombre occidental y una mujer asiática, más recientemente, con el incremento de la migración laboral interregional, se está registrando un notable aumento de los matrimonios entre personas de distintos países asiáticos. J apón, Taiwán, Corea del Sur o Singapur, por ej emplo, están registrando cifras record de matrimonios internacionales a lo largo de la última década (Siddiqui, 2008). Además se está produciendo un incremento de las agencias matrimoniales internacionales y del "mercado de novias a distancia" (empleando las nuevas tecnologías de la comunicación, especialmente internet), lo que está dando lugar a crecientes irregularidades en la migración por matrimonio, y está provocando un mayor

3 Es importante no confundir el tráfico y la trata de personas, conceptos que frecuentemente se presentan unidos y que tienen como parte integrante fundamental los movimientos migratorios, pero que son diferentes. La trata es un delito que atenta contra los derechos de la persona, mientras que el tráfico ilícito de personas es un delito que atenta contra el Estado (Cortés, 2006). 


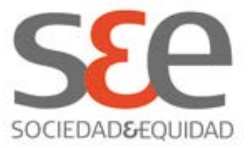

número de mujeres victimizadas en este proceso (Skrobanek, et.al, 1997; Asis, 2005).

\subsection{Migración internacional en América}

Desde una perspectiva histórica, podemos caracterizar tres grandes momentos en los movimientos migratorios del continente americano. 1) Hasta mediados del siglo XX los países americanos fueron receptores de inmigración transoceánica, procedente fundamentalmente de Europa (Martínez Pizarro, 2008), para convertirse posteriormente en países de emigración, salvo en el caso de EE.UU. y Canadá. 2) Desde la década de los sesenta se inicia una emigración permanente y cada vez más intensa desde los países latinoamericanos y caribeños hacia Norteamérica principalmente (Martínez Pizarro, 2011; Pellegrino, 2003), destino al que se va a unir España y otros países europeos desde la década de los noventa (Izquierdo, et.al., 2002; Ocampo y Martin, 2004; Oim, 2012). 3) Entre los países de América Latina y el Caribe también tiene lugar una migración intraregional que, de magnitud moderada, se mantiene a través del tiempo y muestra una tendencia ascendente, con Argentina, Costa Rica, Venezuela y recientemente Chile como principales países receptores de población inmigrante (Sicremi, 2011: 1-8).

Norteamérica es la región del mundo que acoge a más personas inmigrantes internacionales (50 millones en 2010). Prácticamente una de cada cuatro personas migrantes a nivel mundial reside en esta región (exactamente el $23 \%$ ). $Y$ de ellas, la mitad son mujeres.

Estados Unidos lidera el ranking mundial de países de inmigración, con 42,8 millones de personas procedentes de otros países, representado el 13,5\% de su población total. Se estima que 20,5 millones de estas personas inmigrantes provienen de América Latina y el Caribe, siendo más de la mitad personas originarias de México ${ }^{4}$. Esto no obstante, Estados Unidos cuenta también con un destacado flujo migratorio de entrada procedente de Asia (concretamente de China, Filipinas, India, Vietnam, Pakistán, Irán y Bangladesh, por este orden).

Canadá, por su parte, cuenta con 7,2 millones de personas inmigrantes, cuyo peso relativo $(21,3 \%)$ es notablemente superior al registrado por este colectivo en el país vecino. Canadá, con casi 700 mil personas, es decir, el 11\% del total de su población inmigrante, es el tercer principal destino de la emigración latinoamericana y caribeña, tan sólo por detrás de EE. UU. y España.

\footnotetext{
${ }^{4}$ México es el segundo país del mundo exportador de mano de obra (tan sólo superado por Filipinas). Una de cada tres personas inmigrantes en EE.UU. es mexicana (OIM, 2011).
} 


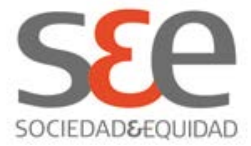

Pero en Canadá el grupo mayoritario de población de origen extranjero (59\%) lo conforman las personas procedentes de Asia (incluido el Medio Oriente), seguido de la población europea (16\%), que ocupa la segunda posición (United Nations, 2012; Sicremi, 2011).

EE.UU. es asimismo el país del mundo con mayor inmigración femenina, con 21, 3 millones de mujeres de origen extranjero establecidas en su territorio. Una de cada cinco mujeres inmigrantes en el mundo reside en este país americano. Canadá, por su parte, se coloca en la cuarta posición de este ranking, con 3,8 millones de mujeres de origen extranjero; cifra muy alejada de la registrada en el país vecino. El stock migratorio en ambos países se muestra bastante equilibrado en función del sexo, aunque en Canadá la tasa de feminización es ligeramente superior a la de EE.UU. (52,2 y 49,8\% respectivamente).

Dado el incremento de las desigualdades como efecto de la mundialización económica (con un impacto mayor en la población femenina), la precarización del empleo, el aumento de la violencia, el recorte de los gastos sociales estatales y la reducción de los servicios públicos (que también afectan de manera especial al trabajo de las mujeres), América Latina y el Caribe constituyen desde hace ya varias décadas dos áreas de emigración, tanto masculina como femenina, dirigida hacia una amplia variedad de destinos, estando los principales de ellos ubicados fuera de la región, entre los países miembros de la OCDE. En el periodo comprendido entre el año 2003 y el 2009, casi 950 mil personas emigraron por año, dirigiéndose la mitad de ellas hacia Estados Unidos y otra cuarta parte hacia España. La gran mayoría de las personas migrantes originarias de América Central y el Caribe, con México a la cabeza, se han dirigido hacia EE.UU., mientras que las procedentes de América del Sur se han marchado fundamentalmente hacia Europa (Oim, 2012), siendo España el principal destino, seguido de países como Italia, Francia o Portugal. En el caso de Brasil, Colombia y Perú, la población emigrante se ha repartido de forma casi igual entre Estados Unidos y Europa (Yépez y Herrera, 2007). La población emigrante de Chile o Argentina también tiene una presencia significativa en otros destinos como Australia e Israel. En el año 2010, más de tres millones de personas originarias de América Latina y el Caribe vivían fuera de la región (Sicremi, 2011).

España, segundo destino de una migración cada vez más diversa procedente de esta región del continente americano, a la que le unen lazos históricos, culturales y lingüísticos, ha registrado un considerable incremento en sus afluencias a lo largo de las dos últimas décadas: Si en el año 1991 eran aproximadamente 210.000 las personas residentes en España nacidas en América Latina, su cifra alcanza ya casi los 2,5 millones a comienzos del 2012. 


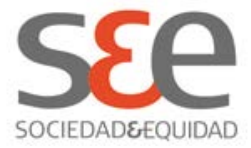

Varias son las razones que, en buena medida, explican este aumento: el cambio de la coyuntura económica en este país europeo, con una creciente demanda laboral hasta fechas recientes (así fuera en sectores de baja cualificación como la agricultura, la construcción o el servicio doméstico), la existencia de un grupo importante de descendientes de personas migrantes españolas y que nunca perdieron la nacionalidad, la posibilidad de la población procedente de esta región de obtener la nacionalidad española por residencia en un periodo de dos años, el establecimiento de acuerdos bilaterales entre España y países de la región como Colombia (año 2001), Ecuador (año 2001) o República Dominicana (año 2002), el liderazgo de este colectivo en los procesos de regularización extraordinarios llevados a cabo en España durante este periodo ${ }^{5}$ (Martínez Buján, 2003), o el mayor acceso a los permisos de residencia en régimen comunitario (que eliminan la necesidad de un permiso de trabajo o la cláusula de prioridad nacional a la hora acceder al mercado laboral), como resultado de los vínculos familiares mantenidos con la población española o con otras personas que disponen de la ciudadanía comunitaria (Vicente y Setién, 2007).

La población latinoamericana más abundante en España es la ecuatoriana, colombiana y argentina, seguida de la boliviana, peruana, venezolana, brasileña dominicana, cubana, uruguaya y paraguaya (Vicente, 2010; López de Lera y Oso, 2007). Dentro de este flujo migratorio, las mujeres han venido mostrando un destacado protagonismo, encabezando en la mayoría de los casos los proyectos migratorios familiares, dada la creciente demanda laboral en sectores de empleo típicamente femeninos, como el servicio doméstico y los cuidados personales. La presencia de mujeres latinoamericanas, especialmente brasileñas, colombianas y dominicanas, en los distintos ámbitos de la "industria del sexo" también ha registrado un notable aumento en las últimas décadas (Azize, 2004; Agustín, 2004).

El predominio femenino es marcado entre la población migrante procedente de Paraguay (70\%), Brasil (64\%), Rep. Dominicana $(61 \%)$, Bolivia (59\%), Colombia (58\%), México (57\%), Cuba (55\%), Perú o Venezuela (54\%) (INE, 2013). Y ello a pesar de que algunos colectivos, principalmente los que cuentan con mayor antigüedad, como es el caso del colombiano o el dominicano, han mostrado una tendencia a su masculinización con el paso de los años, como

\footnotetext{
${ }^{5}$ A pesar de todo ello, la presencia de población latinoamericana indocumentada en España ha sido muy notable, especialmente en el primer quinquenio del siglo XXI. Más de la mitad (51\%) de las personas latinoamericanas residentes en España en el 2004 carecían del preceptivo permiso para trabajar y/o residir en este país, porcentaje que era mucho más elevado entre algunos colectivos nacionales, como entre la población argentina, colombiana o ecuatoriana (Vicente, 2006).
} 


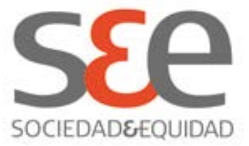

resultado de la reagrupación familiar llevada a cabo por las mujeres pioneras del proceso migratorio desde estos orígenes, las regulaciones políticas o de ciertos cambios en las estrategias empresariales ${ }^{6}$ (Izquierdo, et.al., 2002). Otras nacionalidades latinoamericanas, como la ecuatoriana, la chilena, la argentina o la uruguaya exhiben un mayor equilibro por sexo, si bien las mujeres alcanzan -si no superan ligeramente- las cifras de inmigrantes de los varones.

Desde algunos de estos países se ha dado un flujo migratorio feminizado hacia España y un flujo predominantemente masculino hacia EE.UU. Tal es el caso, por ejemplo, de Colombia (Guarnizo, 2004; García, 2007) o la República Dominicana (Ariza, 2000; Massey, Fischer y Capoferro 2006).

Aparte de EE.UU., Canadá y España, otros destinos de la población migrante latinoamericana son, por orden de presencias, Japón, Italia, Países Bajos, Reino Unido, Francia, Israel, Portugal y Australia (Sicremi, 2011). Las crecientes desigualdades entre origen y destino, la precariedad del empleo y la profundización de las tensiones sociales han dado pie a una sensación generalizada de vulnerabilidad social en la región, ante la que ha cobrado cuerpo una creciente aceptación de la emigración como alternativa para enfrentar las difíciles condiciones de vida (Ocampo y Martin, 2004).

La emigración hacia Japón, creciente desde la década de los noventa, está conformada principalmente por varones brasileños y peruanos. La población caribeña, por su parte, se dirige especialmente hacia Holanda e Inglaterra, países a los que les unen vínculos coloniales, mientras que la población sudamericana que no se dirige a España prefiere otros destinos europeos como son Italia, Francia o Portugal (Martínez Pizarro, 2008).

La emigración continúa siendo, por tanto, la principal característica migratoria de la región conformada por América Latina y el Caribe, a pesar de la disminución de los flujos registrada como consecuencia de la crisis económica internacional que se vive desde el año 2008 y de los diversos programas e incentivos al retorno puestos en marcha por países como EE. UU. 0 España con el objeto de fomentar el regreso al país de origen de la población inmigrante, ante la desfavorable coyuntura económica que atraviesan estos países.

Por otra parte, los principales países receptores de la región latinoamericana -Argentina, Brasil y Venezuela-, también han registrado a lo

\footnotetext{
${ }^{6}$ Como en el caso de la agricultura o la construcción, sectores en los que con el cambio del siglo se van a emplear más trabajadores latinoamericanos, ecuatorianos principalmente, con el objeto de reducir la dependencia de la mano de obra marroquí.
} 


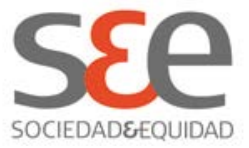

largo de los últimos años un saldo migratorio negativo, debido a las importantes salidas de nacionales que, resultado de la situación económica y/ o política en su país de origen han decidido emigrar en busca de un futuro más prometedor. Esto no obstante, la migración intrarregional en esta parte del continente americano tampoco es nueva, y sí está adquiriendo cada vez una importancia mayor (Sicremi, 2011). Además, algunos países de América Latina como Brasil, Argentina, Chile o Uruguay están comenzando a recibir nuevos flujos migratorios desde fuera de la región, concretamente desde el África Subsahariana, como también los está recibiendo Ecuador desde el Sur de Asia (OIM, 2011: 72-73).

La población migrante internacional residente en un país latinoamericano o caribeño viene mostrando ya desde hace años una gran paridad de género, a pesar de la heterogénea composición de las diversas corrientes migratorias (Martínez Pizarro, 2003). Ésta ha sido la primera región en desarrollo en alcanzar la paridad en el número de mujeres y hombres migrantes, tendencia que se registraba ya a comienzos de la década de los años noventa y que los últimos datos disponibles siguen confirmando.

\subsection{Migración internacional en Europa}

Los flujos migratorios hacia los distintos países europeos han registrado notables cambios a lo largo de las últimas décadas. Frente a la apertura a la inmigración laboral registrada tras la II Guerra Mundial en países como Alemania, Francia o el Reino Unido, otros países europeos como Irlanda, España - Italia continuaban siendo entonces países de emigración. A partir de la década de los años setenta, no obstante, se va a iniciar una nueva etapa, con mayores trabas y controles a la entrada de población inmigrante trabajadora ${ }^{7}$ a estos tradicionales países de recepción ${ }^{8}$, aunque permanece abierta la puerta a la reagrupación familiar realizada por personas ya establecidas en estas sociedades de acogida. Por otra parte, coincidiendo con este cierre de fronteras, los tradicionales países emisores de emigrantes del Sur de Europa van a ir experimentando una mejora en su situación económica y/ o política, así como una reducción en las salidas de su población nacional en busca de

\footnotetext{
7 Restricciones a la entrada de inmigrantes que, aunque redactadas en términos aparentemente neutrales desde el punto de vista del género, han afectado especialmente a las mujeres, reforzando su dependencia de sus cónyuges varones y dificultando su acceso a un status independiente desde el punto de vista legal y social (Kofman y Sales, 1998).

${ }^{8}$ Lo cual no quiere decir que no se produzca inmigración laboral, especialmente abierta a la llegada de personas altamente cualificadas, al igual que ocurre en otros lugares como Canadá, EE.UU. o Australia.
} 


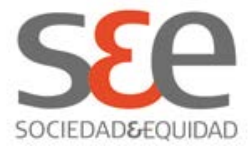

mejores oportunidades en el extranjero, para convertirse ya más recientemente en países de inmigración, tanto de población originaria de otros países europeos como llegada desde otros continentes (Blanco, 2000).

A la hora de analizar los flujos migratorios hacia los distintos países europeos es preciso tener en cuenta, además, el proceso de construcción de la Unión Europea, que desde las últimas décadas del siglo XX ha dado como resultado un espacio en el que se han ido eliminando las trabas a la movilidad de personas en su interior, al tiempo que se han venido reforzando las fronteras exteriores. Así, se va a establecer una clara distinción entre la inmigración no comunitaria (personas que no son ciudadanas de ningún país de la Unión Europea) y la inmigración comunitaria (procedente de un Estado miembro y residente en otro dentro de la Unión Europea ${ }^{9}$ ). Esta última no va a experimentar las restricciones en el número de llegadas, duración de la estancia o acceso al mercado de trabajo que sí se aplican a la población procedente de terceros países, salvo las restricciones temporales al acceso al mercado de trabajo impuestas en el año 2004 a las personas originarias de los nuevos Estados miembros, con motivo de la mayor ampliación de la Europa comunitaria que tuvo lugar entonces (pasando de 15 a 25 países) y a la posterior incorporación en el año 2007 de otros dos países más -Rumanía y Bulgaria. Si bien las distancias que separan a la población nacional de un país de la Unión con las demás personas con ciudadanía europea parecen estrecharse cada vez más, la distancia entre éstas y las personas originarias de terceros países se están acentuando de forma notable.

En este contexto podemos señalar que en la actualidad residen en alguno de los países de la Unión Europea 48,9 millones de personas de origen extranjero, lo que viene a representar el $9,7 \%$ de la población total. De ellas, aproximadamente dos terceras partes (32,4 millones) han nacido fuera de la Unión Europea, mientras que la tercera parte restante (16,5 millones) han nacido en alguno de los otros países de la Europa comunitaria (Vasileva, 2012).

Tres cuartas partes de la población inmigrante residente en la Unión Europea se encuentra en Alemania (9,8 millones), Francia ( 7,3 millones), Reino Unido (7,2 millones), España (6,7 millones) o Italia (5,4 millones). En términos relativos, la presencia de población inmigrante en España (14,3\%) supera a la media comunitaria $(9,7 \%$ y a la registrada en los otros países con una más larga historia de inmigración, como es el caso de Alemania (12\%), Reino Unido $(11,6)$ o Francia (11,2\%). La mayoría de la población inmigrante residente en estos países procede de fuera de la Unión Europea. Únicamente cinco de los

\footnotetext{
${ }^{9}$ También podrán disfrutar del estatuto comunitario las personas casadas cuyo cónyuge sea una persona ciudadana de uno de los Estados miembros de la Unión Europea.
} 


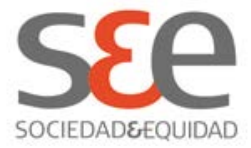

veintisiete estados comunitarios (Luxemburgo, Irlanda, Hungría, Chipre y Malta) cuentan con más población inmigrante originaria de otro país de la Unión Europea que de fuera de la misma (Vasileva, 2012).

Las principales nacionalidades europeas de origen de la población emigrante residente en otro país comunitario son, por este orden, la rumana, la polaca, la italiana, la portuguesa, la inglesa o la alemana. La población no comunitaria residente en alguno de los países de la Unión procede en su mayoría de Turquía, Marruecos, Albania o China. Algunos estados miembros son, al mismo tiempo, importantes países de destino y de origen de población migrante en el seno de la Unión Europea. Tal es el caso, por ejemplo, de Italia, el Reino Unido y Alemania (Eurostat, 2011).

Los países europeos presentan, no obstante, diferencias reseñables en cuanto al origen de la población extranjera residente en su territorio. La migración refleja a menudo la proximidad geográfica o los vínculos históricos, culturales, religiosos y lingüísticos entre los países emisores y receptores, así como el aumento en las oportunidades de empleo en las sociedades de destino. Así se refleja en los flujos migratorios que desde la mitad del siglo pasado vinculan al Caribe o a la India y Pakistán con el Reino Unido (Eurostat, 2011), o a Argelia y Marruecos con Francia (Bokbot y Faleh, 2010). Y, ya más recientemente, a la población emigrante latinoamericana con España preferentemente, pero también con Italia, o a la población brasileña con Portugal (Yépez y Herrera, 2007). En otros casos, en cambio, han sido los acuerdos internacionales entre países necesitados de mano de obra, por una parte, y países con altos niveles de desempleo, por otra, lo que han dado origen a flujos migratorios tan importantes como el de la población turca hacia Alemania (Pérez Díaz, et.al., 2004).

A pesar de la creciente diversificación de orígenes que caracteriza la etapa migratoria actual, Alemania acoge al $75 \%$ de la población turca residente en la Unión Europea, mientras que Italia y España acogen al 78\% de la población migrante de origen rumano. El $88 \%$ de la población marroquí reside en España, Francia o Italia, y el $64 \%$ de las personas procedentes de Polonia reside en el Reino Unido o Alemania. La población albanesa, por su parte, se concentra principalmente en Grecia o Italia, mientras que la mitad de la población portuguesa residente en otro país comunitario diferente al de origen se encuentra en Francia (Vasileva, 2012).

Durante la primera década del siglo XXI, la inmigración registrada en los distintos países de la Unión Europea no ha dejado de crecer, si bien lo ha hecho a distinto ritmo a lo largo de este periodo. Durante la primera mitad de la década el crecimiento fue muy intenso, alcanzando un punto álgido de llegadas en el año 2007. A partir de entonces, y como resultado de la crisis económica, 


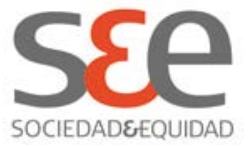

las entradas se han visto en cierta medida reducidas, si bien la población migrante en su conjunto no ha disminuido (Eurostat, 2011). La mala situación económica en muchos lugares emisores así como los procesos de reagrupación familiar explican este crecimiento, así sea más moderado, de la población inmigrante en Europa. Y todo ello, a pesar de la reducción de la inmigración laboral y de los programas de incentivos para el retorno diseñados en este contexto de crisis. Los efectos de la recesión económica se han dejado sentir especialmente en los países del Sur de Europa y en Irlanda.

Desde la perspectiva de género podemos señalar que las crecientes barreras a la inmigración laboral impuestas tras la crisis del petróleo de los años setenta y la mayor apertura a la reagrupación familiar desde entonces van a favorecer la visibilización de la inmigración femenina en los tradicionales países receptores de la Europa Occidental, como son Alemania, Bélgica, Francia o el Reino Unido, aunque sea con un estatuto dependiente de la persona reagrupante (Morokvasic, 1991). Pero, a pesar de la opinión ampliamente compartida de que desde mediados de la década de los setenta la migración femenina pasó a ser dominante en Europa, como consecuencia de los crecientes obstáculos a la inmigración laboral impuestos por parte de los países receptores, la oferta de incentivos para estimular el regreso definitivo a quienes deseasen volver a su país de origen y la mayor apertura a la reagrupación familiar (convertida en la principal vía de entrada), los cambios registrados en la composición por sexo de la población inmigrante no fueron tan importantes como frecuentemente se sugiere (Zlotnik, 1995). Cierto es por otra parte que, si tenemos en cuenta las estadísticas de población extranjera y las de población nacionalizada de origen extranjero en los países europeos que of recen estos datos, el peso de las naturalizaciones es mayor entre las mujeres que entre los hombres, lo que explica en parte la menor representación femenina entre el colectivo de personas extranjeras en algunos de estos países tradicionalmente receptores de inmigración.

Ahora bien, desde la década de los ochenta y, sobre todo de los noventa, se va a producir una feminización del discurso migratorio (Oso, 1998: 42), de modo que las mujeres van a ir adquiriendo un creciente protagonismo, primero como migrantes reagrupadas $y$, posteriormente, también como migrantes autónomas en busca de trabajo (Kofman, 1999). A ello también va a contribuir la migración procedente de la Europa del Este, área geográfica que cuenta con la población emigrante más feminizada (Morokvasic, 2008). Con la desintegración de la antigua URSS y de la exYugoslavia, y con la apertura de fronteras en el bloque comunista, los flujos desde la Europa Oriental se van a incrementar desde la década de los noventa, aunque quizá no con la intensidad en algunos escritos predicha. 


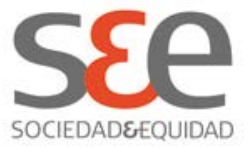

En la actualidad el peso relativo estimado de la inmigración femenina $(52,3 \%)$ es ligeramente superior al de la masculina $(47,7 \%)$, si bien se observan diferencias entre las diferentes regiones europeas: Éste es aproximadamente el porcentaje que representa la población inmigrante femenina en los países del Norte de Europa (53\%), siendo claramente superado en los países de Europa del Este (57\%), mientras que en los países europeos del Sur es ligeramente inferior (50\%), e incluso, como en décadas anteriores, sigue siendo superado por la inmigración masculina en los tradicionales países de inmigración de Europa Occidental (donde ellas representan el $49 \%$ de la población inmigrante) (United Nations, 2012).

Como ya ha quedado apuntado anteriormente, desde la década de los años ochenta y principalmente a lo largo de la década de los noventa, los países del Sur de Europa pasaron a convertirse en países de inmigración (King, 2001; Solé, 2001), recibiendo un importante flujo de población procedente del Norte de África, de muchos lugares de América Latina, de Europa Central y del Este y del Sudeste asiático (Anthias y Lazaridis, 2000).

En este contexto, España, un país de emigración hasta la década de los ochenta, se va a convertir en el tercer país europeo en cuanto a crecimiento de población inmigrante en la década de los noventa, tan sólo por detrás de Finlandia y Grecia. Y ya con el cambio de siglo se va a situar como el principal país de inmigración de la Unión Europea ${ }^{10}$, ranking que ha liderado hasta el año 2007. A partir de entonces el impacto de la crisis económica se está dejando notar, disminuyendo las entradas de población inmigrante. En el 2008 el Estado español pasó a ocupar la segunda posición europea en el número de llegadas, por detrás de Italia; y en el año 2009 la tercera posición, por detrás del Reino Unido y de Italia (OCDE, 2011).

A pesar de que la tasa de feminización de la población inmigrante varía de unos países del Sur de Europa a otros (por ejemplo del 44,6\% de Grecia al $53,1 \%$ de Italia), muchos de los flujos hacia estos países se muestran claramente feminizados, como es el caso de la población procedente de Filipinas y de países latinoamericanos como Colombia, República Dominicana o Brasil. En el caso de Grecia, uno de los países de la cuenca mediterránea con menos inmigración femenina, se observa por ejemplo como algunos grupos nacionales son casi exclusivamente masculinos, como los conformados por las personas originarias de los países árabes o de muchos países asiáticos (a excepción de Filipinas), mientras las personas procedentes de Ucrania 0 Moldavia son, en cambio, en un $70 \%$ mujeres. En Italia, la población de origen magrebí (marroquí, tunecina o egipcia) es mayoritariamente masculina, al igual

${ }^{10} \mathrm{Y}$ el segundo del mundo, tan sólo por detrás de EE.UU. 


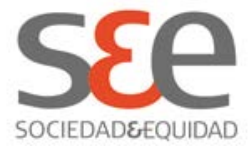

que la llegada desde Sri Lanka, mientras que la procedente de Rumanía, Ucrania, Filipinas, Polonia, Moldavia, Perú o Ecuador muestra una clara mayoría femenina (OCDE, 2011). En España también se registra una clara feminización de algunos colectivos inmigrantes, tales como el brasileño, el colombiano o el boliviano, mientras que otros, como el marroquí, el portugués o el chino, exhiben por su parte un predominio masculino (Ine, 2012). En el modelo migratorio de Portugal, en cambio, predominan los varones en todas las principales nacionalidades de origen, con la única excepción de la brasileña, aunque también en este caso por escaso margen (OCDE, 2011).

Los roles y los puestos asignados a hombres y mujeres migrantes en estas sociedades receptoras son claramente diferentes, explicando esta mayor feminización de determinados colectivos de inmigrantes: la mayoría de las mujeres inmigrantes van a encontrar su nicho de empleo en los sectores más bajos de la escala laboral, peor pagados, menos cualificados, frecuentemente temporales y/ o a tiempo parcial (Koser y Lutz, 1998; Anthias y Lazaridis, 2000; Colectivo loé, 2001). Esto es, en los trabajos considerados típicamente femeninos, que van siendo abandonados por las mujeres autóctonas por ser emblemáticos de la discriminación de género, y que van a ofrecer escasas oportunidades de movilidad laboral o social ascendente. Tal es el caso del servicio doméstico, el sector de los cuidados personales (Andall, 2000; Escrivà, 2000; Anderson, 2000) O la denominada «industria del sexo» (J uliano, 2002; López y Mestre, 2006). A diferencia del tradicional modelo migratorio fordista que durante los años 50's, 60's y 70's empleó a las mujeres inmigrantes en el sector de la industria de los países del Norte de Europa, el modelo postfordista del Sur de Europa va a emplear a las mujeres inmigrantes de forma prioritaria en el sector terciario, en las tareas socialmente menos valoradas del sector servicios (King y Zontini, 2000).

El crecimiento del sector terciario de los sistemas económicos, junto a la segmentación de los mercados de trabajo y al incremento de la flexibilidad y de los sectores informales, van a dar lugar a una serie de nichos laborales reservados de forma particular a mujeres inmigrantes, especialmente en el sector de los servicios de baja cualificación, caracterizados por su precariedad y una escasa protección social y legal (Freedman, 2003). La creciente -aunque todavía relativamente reciente- incorporación de las mujeres autóctonas al trabajo extradoméstico en los países del Sur de Europa, la todavía escasa participación de los hombres en la realización de las tareas del hogar y del cuidado, así como la reducida implicación del Estado en la provisión de políticas sociales con las que hacer frente a esta situación (realidad que se ve agravada en la actual coyuntura de crisis y debilitamiento del Estado de bienestar, con continuos recortes en los servicios sociales ofrecidos), han llevado a un número creciente de familias a recurrir al exterior, concretamente 


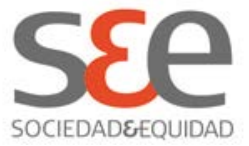

a las mujeres inmigrantes, para la realización de estas labores domésticas y de cuidado de personas dependientes (Parella, 2003; Lutz, 2008; Martínez Buján, 2010). Aproximadamente uno de cada diez hogares en varios países europeos cuenta con personas empleadas en la realización de dichas tareas domésticas y de cuidado; la mayoría de ellas, mujeres (Lutz, 2010). Y ello debido a que las mujeres de clase media de muchas de estas sociedades se incluyen en el denominado por Andall (2000) "paradigma post-feminista", conciliando la vida laboral y familiar mediante la externalización de, al menos, parte del trabajo doméstico, para cuya realización serán empleadas de forma creciente las mujeres inmigrantes. Ahora bien, la creciente presencia de empleadas de hogar, internas o por horas, en los hogares europeos, especialmente de la cuenca mediterránea, va a posponer la discusión del reparto de roles patriarcales en el seno del hogar y de la sociedad (Phizacklea, 1998). Y, de forma paralela, va a poner en evidencia las crecientes desigualdades entre las mujeres -autóctonas e inmigrantes- en estos países (Morokvasic, 2008). La trasferencia del trabajo de cuidado funciona como una cadena global en la que prácticamente participan sólo las mujeres.

Muchas mujeres con altos niveles de formación, incluso con estudios universitarios, van a experimentar con la migración una movilidad social descendente (Vicente, 2006), a pesar de lo cual frecuentemente van a valorar su experiencia migratoria como positiva en la medida en que contribuye a la mejora de las condiciones de vida familiares y a la ampliación de las oportunidades educativas especialmente para sus hijos e hijas. En todo caso, si la llegada de mujeres hacia los países europeos de larga tradición migratoria ha estado tradicionalmente relacionada con el reagrupamiento familiar, la inmigración más reciente, sobre todo hacia el sur de Europa, no se encuentra mayoritariamente en esta categoría.

\section{Para ir concluyendo...}

Ya del simple análisis cuantitativo-descriptivo presentado en este artículo se desprende que los crecientes procesos migratorios a nivel internacional muestran una gran heterogeneidad y complejidad, marcada en buena medida por la variable género. Hombres y mujeres participan en un volumen muy similar en las migraciones internacionales, aunque no siempre coincidan ni los principales países emisores ni receptores, según sea el sexo de las personas migrantes. $Y$ es que las migraciones son procesos generizados.

Desde hace ya tres décadas -periodo en el que comenzamos a contar con algunos datos desagregados por sexo-, la realidad migratoria nos dice que las migraciones internacionales no pueden ser analizadas tomando únicamente como modelo la migración masculina -lo cual todavía ocurre con demasiada frecuencia-, puesto que existen diferentes oportunidades para hombres y para 


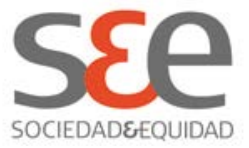

mujeres tanto en los países de origen como de destino, distintas regulaciones de la movilidad humana según la persona migrante sea hombre o mujer, establecidas asimismo desde los Estados y sociedades de emigración como de recepción, o diferentes motivaciones individuales, familiares $\mathrm{y} / \mathrm{o}$ sociales que llevan a las personas migrantes a plantear, según su sexo, proyectos migratorios diferenciados. Profundizando un poco más en este último aspecto, considero además muy importante no confundir la vía de entrada (ej. a través de la reagrupación familiar) o la necesidad de un trabajo remunerado en destino que permita la obtención de los recursos económicos para la supervivencia individual y/o familiar con el/los verdadero(s) motivo(s) migratorio(s), ya que no siempre han de ser coincidentes $y$, de este modo, no llegaremos a ahondar en estos últimos, perdiendo valiosa información en torno a las verdaderas causas de la migración.

Las relaciones de género conforman un rasgo constitutivo de los elementos sociales, económicos y culturales que estructuran la migración, y ésta, asimismo, reconfigura las relaciones de género. Aspecto este último que excede el objetivo del presente artículo, pero que habrá de ser tenido asimismo en cuenta para entender la migración humana en toda su complejidad. $Y$, por supuesto, no se trata de equiparar el concepto de género a la referencia única de las mujeres, sino que el género es una herramienta analítica igualmente relevante para entender la migración masculina (por ello en este artículo se han aludido tanto a flujos y stocks migratorios mayoritariamente masculinos como femeninos).

En estas breves páginas tan sólo se ha tratado de poner en evidencia la importancia de la presencia -diferenciada- de hombres y mujeres en el panorama migratorio internacional, tanto desde el punto de vista de los países emisores como receptores. Un punto de partida que deja numerosos interrogantes, cuya respuesta exige una mayor profundización en la reflexión en torno al género y a la migración internacional en ámbitos geográficos más concretos, y desde diferentes disciplinas y enfoques. $Y$ es que, tal como señala Martín Baró (1998), es importante "que no sean los conceptos los que convoquen a la realidad, sino la realidad la que busque a los conceptos; que no sean las teorías las que definan los problemas de nuestra situación, sino que sean los problemas los que reclamen y, por así decirlo, elijan su propia teorización".

El género juega un papel central en la decisión de emigrar y en la composición de la migración internacional, tiene su reflejo en las políticas migratorias y de acceso a la ciudadanía, en las distintas oportunidades laborales ofrecidas a mujeres y hombres, en la división y organización del trabajo doméstico y de la vida reproductiva, en la autonomía experimentada por las personas migrantes 


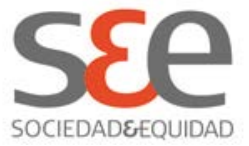

tanto en los países de origen como de destino, en los roles desempeñados por ambos sexos en la esfera pública y privada, etc. El género afecta a todos los aspectos de la migración: sus causas, pautas, procesos e impactos a todos los niveles, incluyendo la propia experiencia migratoria subjetiva de las propias personas migrantes. Éste es, posiblemente, el factor individualmente considerado que más influye en la experiencia migratoria, por lo que queda patente en un simple análisis cuantitativo de los movimientos migratorios internacionales como el aquí presentado. Ahora bien, su reconocimiento no supone sino un primer paso en el análisis, al que habrán de seguir otros que, a nivel macro, meso y micro, permitan explicar su incidencia en los procesos, los discursos y la construcción de identidades que envuelven el diseño de los proyectos y la toma de decisiones migratorias, el reclutamiento, el asentamiento $\mathrm{y} / \mathrm{o}$ el retorno.

\section{Referencias Bibliográficas}

Adepoju, A. (2002), Fostering free movement of persons in West Africa: Achievements, constrains, and prospects for intraregional migration, International Migration, Vol. 40, № 2: 3-28.

Adjei, E. (2006), Impact of female migration on countries of origin: The case of Ghana, Unfpa y Oim (Eds.), Female migrants: Bridging the gaps throughout the life cicle, (pp 47-59). Nueva York: Unfpa, Oim, [http:// www. unfpa.org/ upload/lib pub file/ 658 filename migration. pdf].

Agustín, L.M. (2004), Trabajar en la industria del sexo, y otros tópicos migratorios, Donostia: Gakoa.

Andall, J. (2000), Gender, migration and domestic service, Aldershot: Ashgate.

Anderson, B. (2000), Doing the dirty work? The global politics of domestic labour, Londres: Zed Books.

Anthias, F. y G. Lazaridis, (eds.) (2000), Gender and migration in Southern Europe: Women on the move, Oxford: Berg.

Ariza, M. (2000), Ya no soy la que dejé atrás... Mujeres migrantes en República Dominicana, México, D.F.: Plaza y Valdés.

Asis, M.B. (2005), International migration and prospects for gender equality, Unfpa (Ed.), International migration and the millennium development goals, (pp 113-123). Nueva York, Unfpa.

Azize, Y. (2004), Empujar las fronteras. Mujeres y migración internacional desde América Latina y el Caribe, en R. Osborne (Ed.), Trabajadoras del sexo. 


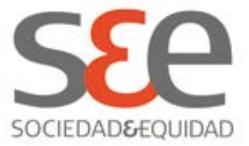

Derechos, migraciones y tráfico en el siglo XXI, (pp 167-180). Barcelona: Bellaterra.

Blanco, C. (2000), Las migraciones contemporáneas. Madrid: Alianza Editorial.

Bokbot, M.M. y A. Faleh (2010), Un siècle d'émigration marocaine vers la France: Aperçu historique, Papeles de Geografía, № 51-52: 55-64.

Chant, S. y S.A. Radcliffe (1992), Migration and development: The importance of gender. En S. Chant (ed.), Gender and migration in developing countries ( $p p$ 1-29). Londres: Belhaven Press, .

Colectivo loé (2001), Mujer, inmigración y trabajo. Madrid: Imserso.

Cortés, D. (2006), El concepto de la trata de personas y su lugar en la agenda migratoria: El caso de los países andinos. En C. Blanco (Ed.), Migraciones: Nuevas movilidades en un mundo en movimiento (160-182). Barcelona: Anthropos.

Engle, L.B. (2004), The world in motion. Short essays on migration and gender. Ginebra: OIM.

Eurostat (2011). Migrants in Europe. A statistical portrait of the first and second generation, Luxemburgo: European Commission. «ttp:/ / ec. europa. eu/ eurostat>.

Freedman, J. (ed.) (2003), Gender and insecurity. Migrant women in Europe, Aldershot: Ashgate, 1-15.

García, M. (2007), Género y remesas. Migración colombiana del AMCO hacia España. Bogotá: INSTRAW-OIM.

Guarnizo, L.E. (2004), La migración transnacional colombiana: Implicaciones teóricas y prácticas. En Colombia nos une (Ed.), Memorias del Seminario sobre migración internacional colombiana y la conformación de comunidades transnacionales ( pp 25-43). Bogotá: Ministerio de Relaciones Exteriores, PNUD y OIM.

Huang, S. y B. Yeoh (2003), The difference gender makes: State policy and contract migrant workers in Singapore, Asian and Pacific Migration Journal, Vol. 12, № 1-2: 75-98.

Hugo, G.J . (1994), Migration and the family. Viena: United Nations.

Instituto Nacional de Estadística (Ine) (1998-2012), Padrón. Población por municipios. Estadística del Padrón Continuo. Disponible en internet: বttp:// www. ine.es>. 


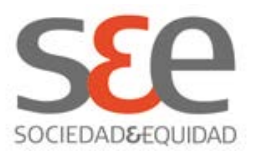

Izquierdo, A. (Et.al.) (2002), Los preferidos del siglo XXI: la inmigración latinoamericana en España, En F.J. García y C. Muriel (Eds), La inmigración en España: contextos y alternativas (237-249). Granada: Laboratorio de Estudios Interculturales.

Juliano, D. (2002), La prostitución: El espejo oscuro. Barcelona: Icaria-Institut Catalá d'Antropologia.

King, R. (Ed.) (2001), The Mediterranean passage: Migration and new cultural encounters in Southern Europe. Liverpool: Liverpool University Press.

King, R. y E. Zontini (2000), The role of gender in the South European immigration model. Papers, № 60: 35-52.

Kofman, E. (1999), Female «birds of passage» a decade later: Gender and immigration in the European Union, International Migration Review, Vol. 33, № 2: 269-299.

Kofman, E. y R. Sales (1998), Migrant women and exclusion in Europe, The European J ournal of Women's Studies, № 5: 381-398.

Koser, K. y H. Lutz (Ed.) (1998). The new migration in Europe. Social constructions and social realities. Londres: MacMillan Press.

López de Lera, D. y L. Oso (2007), La inmigración latinoamericana en España. Tendencias y estado de la cuestión. En I. Yépez y G. Herrera (Eds.), Nuevas migraciones latinoamericanas a Europa. Balances y desafíos (pp 31-67). Quito: Flacso, Obreal, Ucl, Ub.

López, M. y R. Mestre (2006), Trabajo sexual. Reconocer derechos. Valencia: La Burbuja.

Lutz, H. (2010), Gender in the migratory process. Journal of Ethnic and Migration Studies, Vol. 36, № 10: 1647-1663.

Lutz, H. (Ed.) (2008), Migration and domestic work. A European perspective on a global theme. Aldershot: Ashgate.

Martín-Baró, I. (1998), Psicología de la liberación. Madrid: Trotta.

Martínez Buján, R. (2010). Bienestar y cuidados. El oficio del cariño: Mujeres inmigrantes y mayores nativos. Madrid: CSIC.

Martínez Buján, R. (2003). La reciente inmigración latinoamericana a España. Santiago de Chile: CEPAL. 


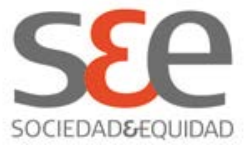

Martínez Pizarro, J orge (Ed.) (2011), Migración internacional en América Latina y el Caribe. Nuevas tendencias, nuevos enfoques. Santiago de Chile: CEPAL.

Martínez Pizarro, Jorge (Coord.) (2008), América Latina y el Caribe: migración internacional, derechos humanos y desarrollo. Santiago de Chile: CEPAL.

Martínez Pizarro, Jorge (2003), El mapa migratorio de América Latina y el Caribe, las muj eres y el género. Santiago de Chile: CEPAL, UNFPA.

Massey, D.S. (Et.al.) (1993), Theories of international migration: A review and appraisal, Population and Development Review, Vol. 19, № 3: 431-466.

Massey, D.S., M.J. Fischer y C. Capoferro (2006), International migration and gender in Latin America: A comparative analysis. International Migration, Vol. 44, № 5: 63-90.

Morokvasic, M. (2008), Crossing borders and shifting boundaries of belonging in post-wall Europe. A gender lens, Disponible en internet: 〈http:// www. migrationonline.cz>.

Morokvasic, M. (1991), Fortress Europe and migrant women. Feminist Review, № 39: 69-84.

Ocampo, J.A. y J. Martin (Coord.) (2004), América Latina y el Caribe en la era global. Colombia: Cepal, Alfaomega.

OCDE (2011), International Migration Outlook: SOPEMI 2011, OECD Publishing. Disponible en internet: 〈http:// dx. doi.org/ 10.1787/ migr outlook-2011-en>.

OIM (2011), Informe sobre las migraciones en el mundo 2011. Comunicar eficazmente sobre la migración. Ginebra: Oim

OIM (Ed.) (2012), Rutas y dinámicas migratorias entre los países de América Latina y el Caribe (ALC), y entre ALC y la Unión Europea. Bélgica: Oim.

Oishi, N. (2005), Women in motion. Globalization, state policies, and labor migration in Asia. Stanford: Stanford University Press.

Oso, L. (1998), La inmigración hacia España de mujeres jefas de hogar. Madrid: Ministerio de Trabajo y Asuntos Sociales.

Parella, S. (2003), Mujer, inmigrante y trabajadora: la triple discriminación. Barcelona: Anthropos.

Parreñas, R.S. (2010), Hacer el amor por un visado: La ciudadanía sexual de las inmigrantes filipinas en Japón. En M. Soronellas (Coord.), Familias en la 


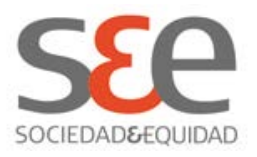

migración. Emociones, solidaridades y obligaciones en el espacio transnacional (pp 97-125). Barcelona: Icaria,.

Pellegrino, A. (2003), La migración internacional en América Latina y el Caribe: Tendencias y perfiles de los migrantes. Santiago de Chile: Cepal, Celade y Bid.

Pérez Díaz, V. (Et.al.) (2004), La inmigración musulmana en Europa. Turcos en Alemania, argelinos en Francia y marroquíes en España. Barcelona: La Caixa.

Phizacklea, A. (1998), Migration and globalization: A feminist perspective. En $\mathrm{K}$. Koser y H. Lutz (Eds.), The new migration in Europe: Social constructions and social realities, (pp 21-38). Londres: MacMillan.

Ryan, J. (2002), Chinese women as transnational migrants: Gender and class in global migration narratives, International Migration, Vol. 40, № 2: 93-116.

Sicremi (2011), Migración internacional en las Américas. Washington: Ocde, Cepal y Oea.

Siddiqui, T. (2008), Migration and gender in Asia. United Nations Expert Group Meeting on International Migration and Development in Asia and The Pacific. Population Division, Bangkok, 20-21 September 2008. Disponible en internet: ४ttp:// www.un.org/ esa/ population/ meetings/EGM_Ittmig_Asia/P06_Siddiqui.pdf>.

Skrobanek, S. (Et.al.) (1997), Tráfico de mujeres. Realidades humanas en el negocio internacional del sexo. Madrid: Narcea.

Solé, C. (Coord.) (2001), El impacto de la inmigración en la economía y en la sociedad receptora. Barcelona: Anthropos.

UN-UNFPA (2006), Estado de la población mundial 2006. Hacia la esperanza: Las mujeres y la migración internacional. Nueva York: United Nations.

United Nations (2012), Trends in International Migrant Stock: Migrants by Destination and Origin (United Nations database, POP/DB/ MIG/Stock/Rev.2012). Disponible en internet: «ttp:// esa. un. org/ MigOrigin/ $>$.

United Nations (2006), 2004 World survey on the role of women in development. Women and international migration. Nueva York: United Nations

Vasileva, K. (2012), Population and social conditions. Eurostat Statistic in focus, Eurostat. Disponible en internet: 


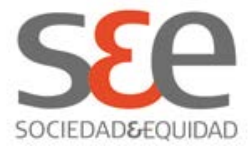

«ttp:/ / epp. eurostat. ec. europa. eu/ portal/ page/ portal/ population/ data/ datab ase>.

Vicente, T.L. (2010), Latin American Immigration to Spain. Network Migration in Europe, Disponible en internet: $\measuredangle t$ ttp:// www. migrationeducation.org>.

Vicente, T.L. (2006), Importancia de los flujos migratorios de mujeres. En C. Blanco (ed.), Migraciones. Nuevas movilidades en un mundo en movimiento (pp 206-233). Barcelona: Anthropos.

Vicente, T.L. y M.L. Setién, M.L. (2007), Actitudes y comportamientos de la población ante los matrimonios mixtos en España. En A. Ibarrola y C.H. Firth (Eds.), Migraciones en un contexto global (pp 129-158). Bilbao: Universidad de Deusto.

Yépez, I. y G. Herrera (Eds.) (2007), Nuevas migraciones latinoamericanas a Europa. Balances y desafíos. Quito: Flacso, Obreal, Ucl, Ub.

Zlotnik, H. (2005), International migration trends since 1980. En Unfta (Ed.) (2005), International migration and the millenium development goals. Selected papers of the Unfta expert group meeting (pp 13-24). Nueva York: Unfta.

Zlotnik, H. (1995), Migration and the family: The female perspective. Asian and Pacific Migration J ournal, Vol. 4, № 2-3: 253-271. 